\title{
Body Mass Index Predicts Insulin Resistance in Survivors of Pediatric Acute Lymphoblastic Leukemia
}

\author{
Stefanie Lowas, MD ${ }^{1,}{ }^{*}$, Suman Malempati, MD1, and Daniel Marks, MD, PhD ${ }^{2}$ \\ ${ }^{1}$ Division of Pediatric Hematology-Oncology, Doernbecher Children's Hospital, Oregon Health \& \\ Science University, Portland, OR \\ 2Division of Pediatric Endocrinology, Doernbecher Children's Hospital, Oregon Health \& Science \\ University, Portland, OR
}

\section{Abstract}

\begin{abstract}
Background-Pediatric acute lymphoblastic leukemia (ALL) therapies have been associated with many late effects, including obesity, hyperglycemia, and insulin resistance. Few data are available linking these abnormalities to specific risk factors present during ALL treatment.
\end{abstract}

\begin{abstract}
Methods-Retrospective cohort study with prospective follow-up. Subjects had been diagnosed with ALL at ages 1-18 years and had been off chemotherapy for $>9$ months. Oral glucose tolerance testing (OGTT) was performed and these results compared to demographic, treatment, and anthropomorphic data from medical records.
\end{abstract}

Results -27 subjects (11 female) were evaluated. Mean ( \pm SD) diagnosis age $5.7 \pm 3.5$ years, mean study age $11.3 \pm 3.7$ years, mean time off therapy $2.8 \pm 1.5$ years. Six subjects had transient hyperglycemia during ALL treatment. At study time, one subject had prediabetes; eight (29.6\%) had insulin resistance. Insulin resistance was not predicted by glucose levels during treatment, cumulative steroid or asparaginase dose, or type of steroid received. Body mass index (BMI) for age correlated significantly with several measures of insulin resistance, including fasting insulin, HOMA index, Matsuda index and insulin AUC ( $p=0.001$ to 0.009). Waist-hip ratio and BMI at ALL diagnosis also correlated with insulin resistance, but these factors' effects could not be separated from BMI at study time.

Conclusions-Variations in ALL therapy and presence of transient hyperglycemia do not appear to increase risk of glucose intolerance or insulin resistance in the first few years after completion of therapy. Elevated BMI strongly predicted insulin resistance in this study, as it does in the general population.

\section{Keywords}

ALL; Hematology/Oncology; Pediatric Endocrinology

\section{INTRODUCTION}

As survival rates from pediatric acute lymphoblastic leukemia (ALL) improve, attention is turning to side effects and late effects of therapy. Many studies have demonstrated increased

Correspondence: Stefanie R. Lowas, MD, Pediatric Hematology-Oncology, 2220 Pierce Ave., 397 PRB, Nashville, TN 37232-6310, Phone: (615) 936-1762, Fax: (615) 936-1767, stefanie.r.lowas@ vanderbilt.edu.

${ }^{*}$ Current address: Division of Hematology/Oncology, Department of Pediatrics, Monroe Carell Jr. Children's Hospital at Vanderbilt, Nashville, TN

Conflicts of interest: none 
rates of obesity in ALL survivors compared to family members or to the general population (1-9). In addition to obesity, other metabolic abnormalities have been identified in ALL survivors, such as dyslipidemia, hyperglycemia, and insulin resistance $(1-3,8,10)$. These abnormalities are linked to the development of cardiovascular disease and type II diabetes, which are leading causes of morbidity and mortality among adults in the United States (11, 12). Aside from obesity itself, the risk factors for developing these metabolic conditions after childhood ALL therapy have not been well defined.

Two chemotherapeutic agents critical to the management of pediatric ALL are corticosteroids and asparaginase. Hyperglycemia is a common side effect of both of these agents, occurring most often during the induction phase of chemotherapy, when they are given in combination in high doses $(13,14)$. This complication, referred to as transient hyperglycemia, is found more frequently in patients with age over 10 years, obesity, Down syndrome, and family history of diabetes. The literature indicates that pediatric ALL patients with transient hyperglycemia, regardless of its severity, recover spontaneously without sequelae $(14,15)$. However, several studies of ALL survivors with various lengths of follow-up have found that many have elevated fasting glucose and/or insulin resistance $(1,2$, $8,16)$. Previous studies did not evaluate whether these later abnormalities of glucose metabolism were linked to transient hyperglycemia. Similarly, it is unknown whether variations in ALL treatment, such as doses of steroids or asparaginase, may predict later alterations in glucose metabolism.

The aim of the current study was to explore potential links between factors present during pediatric ALL therapy and abnormal glucose metabolism after the completion of therapy. This study involved a prospective evaluation of glucose metabolism in recent survivors of pediatric ALL with review of their ALL treatment records. Potential risk factors of interest included presence of hyperglycemia during therapy, cumulative steroid and asparaginase doses and the use of dexamethasone or prednisone in induction. These risk factors, as well as anthropomorphic and demographic variables, were evaluated in relation to the results of the post-treatment glucose testing.

\section{METHODS}

\section{Subjects}

Subjects were identified from the Pediatric Hematology/Oncology patient database at Oregon Health \& Science University (OHSU). The eligible population consisted of children (age $\geq 1$ year and $\leq 18$ years) who were diagnosed with ALL during the years 1997-2004 and had not received chemotherapy in at least 9 months. Subjects received all of their treatment and follow-up care at our institution. Potential subjects who experienced a relapse or second malignancy were excluded. All subjects were treated on open Children's Oncology Group (COG) or Children's Cancer Group (CCG) studies or with the most current standard treatment protocols for pediatric ALL. Subjects identified based on the above criteria were contacted by mail and by telephone and invited to participate in the study. A full description of the study was given to subjects and/or guardians at the study visit and informed consent/child assent was obtained. This study was reviewed and approved by OHSU Cancer Institute and Institutional Review Board.

\section{Oral Glucose Tolerance Testing}

Each subject fasted for at least 12 hours before the testing. Fasting insulin, glucose, and hemoglobin A1C levels were measured. An oral glucose tolerance test (OGTT) was performed in which individuals consumed an oral glucose solution $(1.75 \mathrm{~g} / \mathrm{kg}$ glucose, up to $75 \mathrm{~g}$ maximum). Venous blood samples, from which glucose and insulin levels were 
obtained, were collected at times 15, 30, 60, 90, and 120 minutes after ingestion of the oral solution. Based on these samples, measures of glucose metabolism were calculated. Diabetes was defined as fasting glucose level greater than or equal to $126 \mathrm{mg} / \mathrm{dl}$ or 120 minute glucose level greater than or equal to $200 \mathrm{mg} / \mathrm{dl}$. Prediabetes was defined as fasting glucose level greater than or equal to $100 \mathrm{mg} / \mathrm{dl}$ or 120 minute glucose level greater than or equal to $140 \mathrm{mg} / \mathrm{dl}$. (17) Subjects were defined as having insulin resistance if the fasting insulin level was greater than $15 \mu \mathrm{U} / \mathrm{ml}$ or if the peak insulin level was greater than $150 \mu \mathrm{U} /$ $\mathrm{ml}$. (12) In addition, the OGTT results were used to calculate the insulin and glucose area under the curve (AUC), the HOMA index [fasting insulin $\times$ fasting glucose $\div 22.5$ ], and the Matsuda index $[10000 \div$ square root (fasting glucose $\times$ fasting insulin $\times$ mean glucose $\times$ mean insulin)] $(18,19)$.

\section{Data Collection}

During the study visit, height, weight, and waist and hip circumferences were measured. In addition, the subject or guardian completed a questionnaire about current health status and family history of diabetes. Data regarding the subject's ALL treatment were collected from the medical record on a separate occasion. Information gathered included date of diagnosis, age, height and weight at diagnosis, first recorded serum glucose level, the three highest serum glucose levels recorded during treatment, total cumulative doses of asparaginase, dexamethasone, and/or prednisone, dose (if any) of cranial irradiation, and date of completion of therapy. The first recorded glucose level was of interest as a possible indicator of preexisting glucose metabolism abnormality. The highest recorded glucose level and the average of the three highest recorded glucose levels were used as indicators of hyperglycemia during treatment. In addition, subjects were defined as having transient hyperglycemia if they had greater than two random glucose levels greater than $200 \mathrm{mg} / \mathrm{dl}$. (13) Steroid equivalent doses (using $1 \mathrm{mg} / \mathrm{m}^{2}$ dexamethasone $=6.67 \mathrm{mg} / \mathrm{m}^{2}$ prednisone) were calculated. Due to the change from native L-asparaginase to PEG-asparaginase which occurred during the time period of interest, total asparaginase dose was expressed as number of courses of asparaginase. One course of L-asparaginase refers to either the nine injections of 6000 units $/ \mathrm{m}^{2}$ used in the induction phase of chemotherapy or the six injections of 6000 units $/ \mathrm{m}^{2}$ used in other chemotherapy phases. One course of PEG-asparaginase refers to a single injection of $2500 \mathrm{units} / \mathrm{m}^{2}$. Waist and hip circumferences were used to calculate waist to hip ratio, an indicator of abdominal obesity and a predictor of glucose metabolism abnormalities in children and adults. $(11,12)$ Each subject's body mass index (BMI) was calculated from the height and weight at diagnosis and at study time. Using reference standards from the Centers for Disease Control and Prevention (CDC), BMI percentile for age and gender, and BMI z-score for age and gender were determined. Per current CDC guidelines, subjects with BMI greater than or equal to the $95^{\text {th }}$ percentile for age were defined as overweight and those with BMI greater than or equal to the $85^{\text {th }}$ percentile for age were defined as at risk for overweight. (20)

\section{Statistical Analysis}

All of the data collected were analyzed using SPSS (SPSS versions 15.0 and 16.0 for Windows). Descriptive statistics about the subject population were calculated, including sex, age and BMI at diagnosis and evaluation, presence of family history of diabetes, and time from end of therapy to evaluation. Values obtained from the OGTT were correlated to risk factors obtained from the records review, as well as to demographic and anthropomorphic variables. Linear regression was used to analyze continuous results (such as insulin level) and logistic regression was used to analyze binary results (such as presence or absence of insulin resistance). 


\section{RESULTS}

Twenty-seven subjects ( 11 female) participated in the study. The subjects had been diagnosed with ALL between May 1999 and February 2004. The age at time of ALL diagnosis ranged from 1.3 to 14.9 years (mean 5.7 years, SD 3.5 years) and the age at time of study ranged from 6.5 to 20.3 years (mean 11.3 years, SD 3.7 years). The subjects had been off therapy for an average of 2.8 years (range 0.9 to 6.0 years, SD 1.5 years). See Table I for additional subject information. A total of 64 subjects had been invited to participate in the study. Of those who did not participate, 2 cited other medical problems, 17 cited difficulty scheduling around work/school, 10 were not interested, and 4 did not respond to the invitation. In addition, 2 subjects did not keep study appointments, and 2 agreed to participate but an adequate amount of blood could not be obtained. Specific data could not be collected about the invitees who chose not to participate. From the institutional data which are available, however, the subjects who did participate do appear to be representative of the patients diagnosed with ALL at our institution in 1999-2004. During that time, 25.4\% of newly diagnosed ALL patients had BMIs $\geq 85^{\text {th }}$ percentile for age and gender, compared to $25.9 \%$ of the study subjects. The prevalence of transient hyperglycemia among study subjects was the same $(22.2 \%)$ as that of our institution's ALL patients during that time.

None of the 27 subjects had diabetes based on the OGTT results. One subject just met the criteria for prediabetes, with a fasting glucose level of $101 \mathrm{mg} / \mathrm{dl}$, but this subject's 120 minute glucose level was less than $140 \mathrm{mg} / \mathrm{dl}$. Eight subjects, including the one with prediabetes, (29.6\%) had insulin resistance. Of these, 2 had elevated fasting insulin levels, 2 had elevated peak insulin levels, and 4 had both elevated fasting and peak insulin levels. As a result of these observations, the remainder of the analysis focused on insulin resistance as the glucose metabolism abnormality of interest.

Hyperglycemia during ALL therapy was not a predictor of insulin resistance after therapy. Similarly, there was no correlation between higher cumulative doses of steroids or asparaginase during therapy and later insulin resistance. In addition, there was no statistically significant difference in the presence of insulin resistance between subjects who had received prednisone and those who had received dexamethasone. Table II displays the results of the linear regression analysis of risk factors present during ALL therapy versus various measures of insulin resistance. Similar correlation coefficients and p-values were found whether degree of insulin resistance was measured by fasting insulin, 120 minute insulin, HOMA index, Matsuda index, or insulin AUC. Supplemental Table I demonstrates further details of these analyses.

The impact of demographic, anthropomorphic, and other ALL-related variables on presence of insulin resistance was explored. Supplemental Table I contains details of these results. There was no statistically significant correlation between insulin resistance and gender, age at ALL diagnosis, ALL risk category (standard versus high), age at study time, or time off therapy. Subjects who received cranial irradiation were not more likely to have insulin resistance than those who did not. Family history of diabetes was a significant predictor of insulin resistance based on the Matsuda index, but not the other measures of insulin resistance.

Elevated BMI for age at the time of study was a strong, consistent predictor of insulin resistance. For overweight subjects, the odds of having insulin resistance, either elevated fasting or peak insulin level, was 59.5 times that of the non-overweight subjects (see Table III, 95\% CI 4.6-767.2). All of the subjects with fasting insulin $>15 \mu \mathrm{U} / \mathrm{ml}$ and all but one of those with peak insulin $>150 \mu \mathrm{U} / \mathrm{ml}$ were overweight. Notably, the one subject with elevated fasting glucose was overweight and had insulin resistance as well. For subjects with 
$\mathrm{BMI}>85^{\text {th }}$ percentile for age (overweight or at risk for overweight), the odds of having insulin resistance was 9.6 times that of those with BMI $<85^{\text {th }}$ percentile for age $(95 \%$ CI 1.0-94.5). BMI z-score at study time significantly correlated with fasting and 120 minute insulin levels, HOMA index, Matsuda index, and insulin area under the curve, with p-values ranging from 0.001 to 0.009 (see Table IV and Figure 1). The correlation between BMI zscore at study time and the peak insulin level approached significance $(\mathrm{p}=0.056)$. Similarly, waist to hip ratio correlated with insulin resistance (Table IV). However, BMI z-score was a significant predictor of waist to hip ratio, so it is difficult to separate the effects of these two anthropomorphic measures. The only risk factor present during ALL therapy which was consistently associated with insulin resistance was BMI z-score at the time of ALL diagnosis (Table IV). All of the subjects who were overweight or at risk for overweight at diagnosis remained so at study time. Therefore, as with waist to hip ratio, the effects of BMI at diagnosis and at study time on insulin resistance could not be separated.

A multivariate analysis was performed using BMI z-score at study time, total steroid dose and type, number of asparaginase courses, and presence of transient hyperglycemia as predictive variables for insulin resistance. While limited by the small number of subjects, this analysis demonstrated that BMI z-score was still the only significant predictor of insulin resistance. This remained true whether insulin resistance was expressed as fasting insulin level, HOMA index, or Matsuda index (data not shown). In addition, bivariate analyses were performed to evaluate each of the treatment-related risk factors while controlling for BMI zscore. Again, BMI z-score was significant while the other factors were not (data not shown).

\section{DISCUSSION}

There is a growing body of evidence documenting the presence of abnormalities of glucose metabolism in survivors of pediatric ALL. $(1-3,16,21)$ These abnormalities are well known in the general population to precede type 2 diabetes and cardiovascular disease. $(11,12,17)$ As more pediatric ALL patients become young adult survivors, attending to these adultonset diseases has become a more prominent concern for their medical providers. In addressing these long term health issues, one starting point is to evaluate them in light of potential risk factors present at the time of ALL therapy. Previous studies which have involved measures of glucose metabolism in ALL survivors have not correlated these measurements with treatment-related factors other than presence or absence of cranial irradiation. $(3,21)$ The goal of the current study was to identify treatment-related factors which could be used as predictors for later abnormalities of glucose metabolism. If such predictors were found, they would indicate which ALL survivors are at the highest risk for these abnormalities and hence need the closest screening and follow-up.

Of the chemotherapeutic agents used to treat pediatric ALL, corticosteroids and asparaginase are the most likely to result in glucose metabolism abnormalities. Hyperglycemia has long been recognized as an acute side effect of both of these medications. $(14,15,22)$ In vitro and in vivo studies have demonstrated that steroids cause dose-dependent increase in insulin resistance with decreased glucose uptake into tissues, as well as increased hepatic gluconeogenesis. $(23,24)$ Patients who receive steroids for malignant or inflammatory conditions can develop dose-dependent hyperglycemia and insulin resistance, which usually resolve when the steroids are discontinued. $(15,23,24)$ However, some have suggested that there may be a lasting hyperglycemic effect on patients with or predisposed to diabetes. (23) In contrast to what would be expected based on these mechanisms, the insulin resistance found in our study did not correlate with the total doses of steroids received by the subjects. 
The hyperglycemic effect of asparaginase results from a dose-dependent decrease in insulin secretion from pancreatic islet cells. $(15,22)$ At higher concentrations in vitro, asparaginase has been demonstrated to cause irreversible alteration of asparagine in the cells and/or islet cell death. $(25,26)$ Based on these mechanisms, prolonged abnormalities of glucose metabolism, manifested as hyperglycemia and hypoinsulinemia would be expected in patients who have received asparaginase therapy. Mohn et al. previously studied ALL survivors and found decreased insulin secretion in glucose tolerance testing. $(16,27)$ In contrast, subjects in the current study were found to be hyperinsulinemic rather than hypoinsulinemic. One cause for this difference may be that Mohn et al. excluded overweight children from their analysis, while overweight was the most significant predictor of abnormalities of glucose metabolism in the current study.

The only significant, consistent predictor of insulin resistance in the subjects in this study was BMI percentile for age and gender at the time of study. The odds of finding insulin resistance in overweight subjects were 59.5 times that of the non-overweight subjects.

Similar correlations were found with waist to hip ratio and with BMI for age at the time of ALL diagnosis, however these predictors could not be separated from BMI at study time. Interestingly, while several (22.2\%) of those in this study had transient hyperglycemia during ALL treatment, which has been associated with overweight $(13,14)$, this acute side effect did not predict later insulin resistance. The correlation between BMI for age with insulin resistance in this study reflects the trends already well documented in the general population. $(11,12,17)$

The development of insulin resistance in children and adults is multifactorial, and not all of the mechanisms involved have been clearly delineated. However, as was found in the current study, it is known that adiposity, particularly abdominal adiposity, is associated with increased insulin resistance. $(12,17)$ Family history of type 2 diabetes is also associated with insulin resistance, but this was not definitively found in the current study. It is also known that type 2 diabetes develops once insulin secretion is no longer high enough to compensate for the degree of insulin resistance present. $(12,17)$ Unfortunately, the time needed to progress from insulin resistance to diabetes is unknown. It is possible that this progression may occur more quickly in ALL survivors due to the effects of asparaginase on pancreatic insulin secretion.

This study was significantly limited by its size; 27 subjects were studied instead of the 40 or 50 originally planned. One reason for this was the difficulty in subject recruitment, particularly of adolescents. This may have also led to some selection bias. For example, several parents of subjects stated that they were interested in the study because of its focus on blood glucose. As a result, these individuals chose to take the time to participate, while others decided that the study would not be important enough to miss extra work or school. Additional limitations include the variation in age and time off therapy of the subjects. These, too, were related to the few adolescents who participated, but did not appear to have an effect on results, since age and time off therapy were not associated with insulin resistance. A final challenge in the analysis of the data in this study was the fact that some subjects received L-asparaginase, some received PEG-asparaginase, and some received both. There is not a clear conversion factor for the doses of these medications, as exists for steroids. Therefore, this factor was expressed as number of courses of asparaginase rather than a unit dose equivalent.

The late effects of chemotherapy on glucose metabolism still deserve further research. The purpose of the current study was to discover whether variations the treatment course of ALL were associated with later glucose metabolism abnormalities, independent of overweight. Most of the limitations mentioned above would be ameliorated by a large multi-institutional 
study. It is possible that, with enough subjects, an analysis controlling for the effect of BMI may be performed. This could discover late effects of the steroids and asparaginase that were not found here. Meanwhile, ALL survivors will still be closely monitored for late effects, as they are now. With the goal of optimizing the overall health of survivors, it may be wise for pediatric oncologists to follow the lead of general pediatricians and screen overweight patients for abnormalities of glucose metabolism with fasting glucose levels. $(17,28)$

\section{Supplementary Material}

Refer to Web version on PubMed Central for supplementary material.

\section{Acknowledgments}

This publication was made possible with support from the Oregon Clinical and Translational Research Institute (OCTRI), grant number UL1 RR024140 from the National Center for Research Resources (NCRR), a component of the National Institutes of Health (NIH), and NIH Roadmap for Medical Research. Dr. Lowas' research was sponsored in part by a Tartar Trust grant from the OHSU foundation.

\section{References}

1. Kourti M, Tragiannidis A, Makedou A, et al. Metabolic syndrome in children and adolescents with acute lymphoblastic leukemia after the completion of chemotherapy. J Pediatr Hematol Oncol. 2005; 27(9):499-501. [PubMed: 16189445]

2. Jarfelt M, Lannering B, Bosaeus I, et al. Body composition in young adult survivors of childhood acute lymphoblastic leukaemia. Eur J Endocrinol. 2005; 153(1):81-9. [PubMed: 15994749]

3. Oeffinger KC, Buchanan GR, Eshelman DA, et al. Cardiovascular risk factors in young adult survivors of childhood acute lymphoblastic leukemia. J Pediatr Hematol Oncol. 2001; 23(7):42430. [PubMed: 11878576]

4. Rogers PC, Meacham LR, Oeffinger KC, et al. Obesity in pediatric oncology. Pediatr Blood Cancer. 2005; 45(7):881-91. [PubMed: 16035086]

5. Van Dongen-Melman JE, Hokken-Koelega AC, Hahlen K, et al. Obesity after successful treatment of acute lymphoblastic leukemia in childhood. Pediatr Res. 1995; 38(1):86-90. [PubMed: 7478803]

6. Didi M, Didcock E, Davies HA, et al. High incidence of obesity in young adults after treatment of acute lymphoblastic leukemia in childhood. J Pediatr. 1995; 127(1):63-7. [PubMed: 7608813]

7. Shaw MP, Bath LE, Duff J, et al. Obesity in leukemia survivors: The familial contribution. Pediatr Hematol Oncol. 2000; 17(3):231-7. [PubMed: 10779989]

8. Talvensaari KK, Lanning M, Tapanainen P, Knip M. Long-term survivors of childhood cancer have an increased risk of manifesting the metabolic syndrome. J Clin Endocrinol Metab. 1996; 81(8): 3051-5. [PubMed: 8768873]

9. Warner JT, Evans WD, Webb DK, Gregory JW. Body composition of long-term survivors of acute lymphoblastic leukaemia. Med Pediatr Oncol. 2002; 38(3):165-72. [PubMed: 11836715]

10. Link K, Moell C, Garwicz S, et al. Growth hormone deficiency predicts cardiovascular risk in young adults treated for acute lymphoblastic leukemia in childhood. J Clin Endocrinol Metab. 2004; 89(10):5003-12. [PubMed: 15472198]

11. Molnar D. The prevalence of the metabolic syndrome and type 2 diabetes mellitus in children and adolescents. Int J Obes Relat Metab Disord. 2004; 28(Suppl 3):S70-4. [PubMed: 15543223]

12. Ten S, Maclaren N. Insulin resistance syndrome in children. J Clin Endocrinol Metab. 2004; 89(6): 2526-39. [PubMed: 15181020]

13. Baillargeon J, Langevin AM, Mullins J, et al. Transient hyperglycemia in hispanic children with acute lymphoblastic leukemia. Pediatr Blood Cancer. 2005; 45(7):960-3. [PubMed: 15700246]

14. Pui CH, Burghen GA, Bowman WP, Aur RJ. Risk factors for hyperglycemia in children with leukemia receiving L-asparaginase and prednisone. J Pediatr. 1981; 99(1):46-50. [PubMed: 6454771] 
15. Howard SC, Pui CH. Endocrine complications in pediatric patients with acute lymphoblastic leukemia. Blood Rev. 2002; 16(4):225-43. [PubMed: 12350366]

16. Mohn A, Di Marzio A, Capanna R, et al. Persistence of impaired pancreatic beta-cell function in children treated for acute lymphoblastic leukaemia. Lancet. 2004; 363(9403):127-8. [PubMed: 14726167]

17. Kaufman FR. Type 2 diabetes in children and youth. Endocrinol Metab Clin North Am. 2005; 34(3):659, 76, ix-x. [PubMed: 16085165]

18. Matthews DR, Hosker JP, Rudenski AS, et al. Homeostasis model assessment: Insulin resistance and beta-cell function from fasting plasma glucose and insulin concentrations in man. Diabetologia. 1985; 28(7):412-9. [PubMed: 3899825]

19. Matsuda M, DeFronzo RA. Insulin sensitivity indices obtained from oral glucose tolerance testing: Comparison with the euglycemic insulin clamp. Diabetes Care. 1999; 22(9):1462-70. [PubMed: 10480510]

20. Ogden CL, Flegal KM, Carroll MD, Johnson CL. Prevalence and trends in overweight among US children and adolescents, 1999-2000. JAMA. 2002; 288(14):1728-32. [PubMed: 12365956]

21. Gurney JG, Ness KK, Sibley SD, et al. Metabolic syndrome and growth hormone deficiency in adult survivors of childhood acute lymphoblastic leukemia. Cancer. 2006; 107(6):1303-12. [PubMed: 16894525]

22. Cetin M, Yetgin S, Kara A, et al. Hyperglycemia, ketoacidosis and other complications of Lasparaginase in children with acute lymphoblastic leukemia. J Med. 1994; 25(3-4):219-29. [PubMed: 7996065]

23. Olefsky JM, Kimmerling G. Effects of glucocorticoids on carbohydrate metabolism. Am J Med Sci. 1976; 271(2):202-10. [PubMed: 178180]

24. Chan JC, Cockram CS, Critchley JA. Drug-induced disorders of glucose metabolism. Mechanisms and management. Drug Saf. 1996; 15(2):135-57. [PubMed: 8884164]

25. Clausen N, Nielsen JH. Direct long-term effects of L-asparaginase on rat and human pancreatic islets. Pediatr Res. 1989; 26(2):158-61. [PubMed: 2671905]

26. Pou JM, Cervera T, Perez A, et al. Effect of L-asparaginase on insulin secretion from isolated rat islets of langerhans. Horm Res. 1991; 35(3-4):155-60. [PubMed: 1725285]

27. Mohn A, Di Marzio D, De Berardiniis A, et al. Long-term follow-up of children treated for acute lymphoblastic leukemia and the recovery of beta-cell function. Haematologica. 2006; 91(10): 1424-5. [PubMed: 16963397]

28. Hannon TS, Rao G, Arslanian SA. Childhood obesity and type 2 diabetes mellitus. Pediatrics. 2005; 116:473-480. [PubMed: 16061606] 

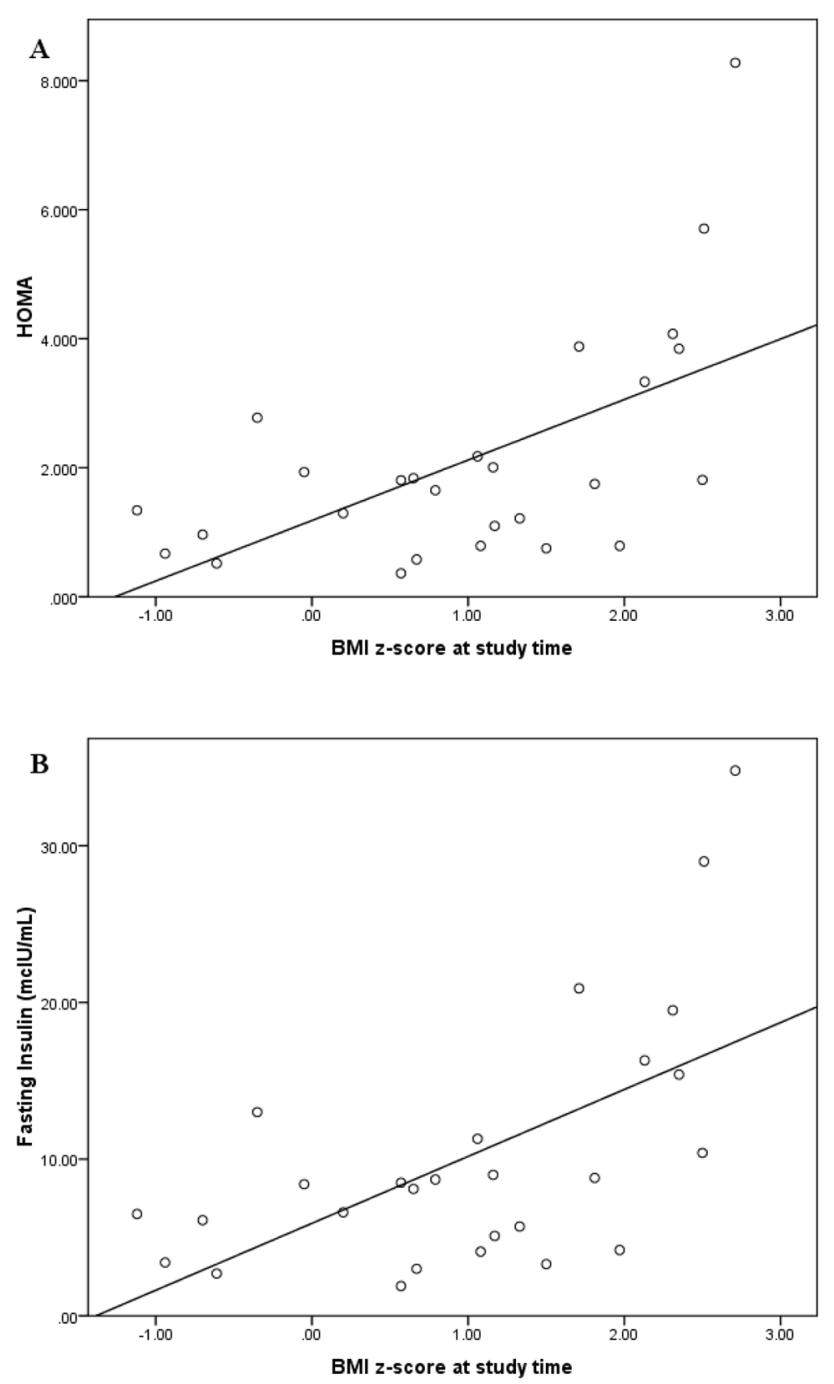

Figure 1.

BMI z-score at study time plotted against HOMA index (A) and fasting insulin (B), with linear regression line shown. Pearson correlation coefficient is 0.588 in A and 0.596 in B. 


\section{Table I}

\section{Subject Demographic and Background Data}

\begin{tabular}{|c|c|}
\hline Characteristic & Number (Percent) \\
\hline \multicolumn{2}{|l|}{ Sex } \\
\hline Female & $11(40.7 \%)$ \\
\hline Male & $16(59.3 \%)$ \\
\hline \multicolumn{2}{|l|}{ Age at diagnosis } \\
\hline$<10$ years & $23(85.2 \%)$ \\
\hline$>10$ years & $4(14.8 \%)$ \\
\hline \multicolumn{2}{|l|}{ BMI category at diagnosis } \\
\hline Overweight $\left(\geq 95^{\text {th }} \text { percentile for age }\right)^{*}$ & $5(18.5 \%)$ \\
\hline Overweight or at risk ( $285^{\text {th }}$ percentile for age) & $7(25.9 \%)$ \\
\hline Average or below average $\left(<85^{\text {th }}\right.$ percentile for age $)$ & $20(74.1 \%)$ \\
\hline \multicolumn{2}{|l|}{ BMI category at time of study } \\
\hline Overweight ( $\geq 95^{\text {th }}$ percentile for age $)^{*}$ & $9(33.3 \%)$ \\
\hline Overweight or at risk ( $\geq 85^{\text {th }}$ percentile for age) & $15(55.6 \%)$ \\
\hline Average or below average $\left(<85^{\text {th }}\right.$ percentile for age $)$ & $12(44.4 \%)$ \\
\hline \multicolumn{2}{|l|}{ Family history of diabetes } \\
\hline Yes & $13(48.1 \%)$ \\
\hline No & $14(51.9 \%)$ \\
\hline \multicolumn{2}{|l|}{ ALL risk category } \\
\hline High & $10(37.0 \%)$ \\
\hline Standard & $17(63.0 \%)$ \\
\hline \multicolumn{2}{|l|}{ Steroid received in induction } \\
\hline Prednisone & $14(51.9 \%)$ \\
\hline Dexamethasone & $13(48.1 \%)$ \\
\hline \multicolumn{2}{|l|}{ Cranial or craniospinal irradiation received } \\
\hline Yes & $3(11.1 \%)$ \\
\hline No & $24(88.9 \%)$ \\
\hline \multicolumn{2}{|l|}{ Transient hyperglycemia during induction } \\
\hline Yes & $6(22.2 \%)$ \\
\hline No & $21(77.8 \%)$ \\
\hline
\end{tabular}

Pediatr Blood Cancer. Author manuscript; available in PMC 2013 October 21. 
Table III

Odds of Insulin Resistance by BMI Category

\begin{tabular}{cccc}
\hline Risk Factor & fasting insulin $>\mathbf{1 5} \boldsymbol{\mu \mathbf { U } / \mathbf { m l }}$ & peak insulin $>\mathbf{1 5 0} \boldsymbol{\mu} \mathbf{U} / \mathbf{m l}$ & fasting or peak insulin resistance \\
\hline Diagnosis BMI $>95^{\text {th }}$ percentile & $40.0(2.9-554.7)^{*}$ & $9.5(1.1-82.7)$ & $\mathrm{N} / \mathrm{A}^{* * *}$ \\
& $\mathrm{p}=0.006$ & $\mathrm{p}=0.041<0.001$ \\
Diagnosis BMI $>85^{\text {th }}$ percentile & $12.0(1.5-97.2)$ & $4.3(0.6-29.5)$ & $14.2(1.8-109.9)$ \\
& $\mathrm{p}=0.020$ & $\mathrm{p}=0.143$ & $\mathrm{p}=0.011$ \\
Study BMI $>95^{\text {th }}$ percentile & $\mathrm{N} / \mathrm{A}^{* *}$ & $21.3(1.9-236.0)$ & $59.5(4.6-767.2)$ \\
& $\mathrm{p}<0.001$ & $\mathrm{p}=0.013$ & $\mathrm{p}=0.002$ \\
Study BMI $>85^{\text {th }}$ percentile & $\mathrm{N} / \mathrm{A}^{* *}$ & $5.5(0.5-55.5)$ & $9.6(1.0-94.5)$ \\
& $\mathrm{p}<0.001$ & $\mathrm{p}=0.148$ & $\mathrm{p}=0.052$ \\
\hline
\end{tabular}

* Odds ratio ( $95 \%$ confidence interval) calculated by logistic regression;

**

Odds ratio could not be calculated because all subjects with fasting insulin resistance had BMI $>95^{\text {th }}$ percentile;

Odds ratio could not be calculated because all of those without insulin resistance had BMI $<95^{\text {th }}$ percentile. 
